\title{
Down-regulation of RASA1 Is Associated with Poor Prognosis in Human Hepatocellular Carcinoma
}

\author{
YAO-LI CHEN ${ }^{1,2,3}$, WEI-CHIEH HUANG ${ }^{4}$, HSIN-LEI YAO ${ }^{5}$, PO-MING CHEN ${ }^{4,6}$, \\ PING-YI LIN ${ }^{2}$, FU-YU FENG ${ }^{7 *}$ and PEI-YI CHU ${ }^{6,8,9 *}$ \\ ${ }^{1}$ School of Medicine, Kaohsiung Medical University, Kaohsiung, Taiwan, R.O.C.; \\ ${ }^{2}$ Department of General Surgery, Changhua Christian Hospital, Changhua, Taiwan, R.O.C.; \\ ${ }^{3}$ Transplantation Center, Third Xiangya Hospital of Central South University, Changsha, P.R. China; \\ ${ }^{4}$ Institute of Molecular and Genomic Medicine, National Health Research Institutes, Zhunan, Taiwan, R.O.C.; \\ ${ }^{5}$ Research Consultant, Richmond, VA, U.S.A.; \\ ${ }^{6}$ Department of Pathology, Show Chwan Memorial Hospital, Changhua, Taiwan, R.O.C.; \\ ${ }^{7}$ Department of Medical Imaging, Show Chwan Memorial Hospital, Changhua, Taiwan, R.O.C.; \\ ${ }^{8}$ School of Medicine, Fu-Jen Catholic University, New Taipei City, Taiwan, R.O.C.; \\ ${ }^{9}$ National Institute of Cancer Research, National Health Research Institutes, Tainan, Taiwan, R.O.C.
}

\begin{abstract}
Background/Aim: RASAl (p120RasGAP), encodes Ras GTPase-activating protein 1 and, is a potent tumor suppressor gene that is frequently inactivated in several human cancer types. However, its precise role in hepatocellular carcinoma (HCC) has been blurred. Materials and Methods: We hypothesized that RASAl plays a crucial role in tumor pathogenesis and progression of HCC. RASAl expression levels were analyzed in 226 cases of HCC by immunohistochemistry. Results: It was found that $38.68 \%$ (41/106) of the high-grade HCC samples and $54.17 \%$ (65/120) of the low-grade HCC samples expressed RASAl protein. The difference between RASAl expression in high-grade and low-grade HCC was statistically significant $(p=0.02)$. Additionally, RASAl high expression was inversely associated with larger tumor size $(p<0.001)$. Although RASAl is known as a tumor suppressor, its role in overall survival (OS) in HCC is unclear. Kaplan-Meier survival analysis showed that patients with low level of RASAl
\end{abstract}

\footnotetext{
*These Authors contributed equally to this study.

Correspondence to: Fu-Yu Feng, MD, Department of Medical Imaging, Show Chwan Memorial Hospital, No.542, Sec.1, ChungShang Road, Changhua City, Changhua County, 50008, Taiwan, R.O.C. Tel: +88647256166 ext 66088 , Fax: +886 47233190, email: fieyel@gmail.com and Pei-Yi Chu, MD, Ph.D., Department of Pathology, Show Chwan Memorial Hospital, No.542, Sec.1, Chung-Shang Road, Changhua City, Changhua County, 50008, Taiwan, R.O.C. Tel: +886 47256166 ext 81688, Fax: +886 47233190, e-mail: chu.peiy@msa.hinet.net
}

Key Words: Liver cancer, overall survival, RASA1, RAS-GAP. expression correlated with a significantly poorer survival compared to those with high level of RASAl expression. Conclusion: These data support that RASAl could serve as an independent prognostic marker for HCC patients.

Liver cancer, including hepatocellular carcinoma (HCC), is the fifth most common cancer worldwide and the second leading cause of cancer-related deaths worldwide (1). Established risk factor for liver cancer include chronic infection with HBV or HCV, heavy alcohol use, and most probably non-alcoholic fatty liver disease (2). HCC will subsequently lead to elevated angiogenesis and metastasis, which may increase the chemotherapy resistance and be responsible for the poor survival of $\mathrm{HCC}$ patients $(2,3)$. Systemic therapies for $\mathrm{HCC}$ patients are still facing challenges regarding drug resistance and drug toxicity.

RAS is a small GTP binding protein that may act as molecular switches of signaling transduction modulating many aspects of cell development involving cell growth, differentiation and survival (4). RAS cycles are characterized by two different conformations, an inactive GDP-bound and an active GTP-bound state, which are controlled by RAS guanine nucleotide exchange factors (GEF) and RAS GTPase-activating proteins (GAPs) (5). RAS-GAP mediates inactivation of RAS proteins by promoting the intrinsic GTPase activity of RAS. A study indicated that the RasGAPs are negative regulators of Ras and its function as potential tumor suppressors (6). Thus, inactivation of RasGAPs could decrease the risk for tumor progression (7).

In addition, patients with liver cancer have high incidence of relapse after resection (8). Recently, the hypothesis of cancer-initiating cells indicated that they have a high 
potential to metastasize and often cause relapse after treatment (9). Previous studies demonstrate that RAS-GAPs are a suppressor in cancer, which modulates cancer stemness and epithelial-mesenchymal transition (EMT) contributing to relapse $(10,11)$.

RASA1 (Ras GTPase-activating protein 1; also known as p120RasGAP), is the first identified RasGAP protein. Previous studies have indicated that PTP1B dephosphorylates PITX1 to weaken its protein stability and further reduce RASA1 mRNA expression inhibition, suggesting that RASA1 may involved in the suppression of tumor progression (12). RASA1 has been involved in many biological processes including actin filament polymerization, cellular apoptosis, and cell migration (13). Besides, several studies indicated that onco-microRNAs (micorRNA-21 and micorRNA-182) can promote tumor angiogenesis or lymph node metastasis by targeting RASA1 $(14,15)$. Therefore, RASA1 functions as a tumor suppressor in several types of cancer. However, study of the role for RASA1 in HCC is relatively scarce.

The aim of this study was to analyze whether RASA1 expression is related to clinicalpathological characteristics and prognostic significance in HCC. To examine RASA1 expression in human HCC clinical samples, 226 surgically resected HCC tumors were subject to immunohistochemistry analysis. Then it further investigated whether combination of RASA1 expression and clinicopathological parameters cuuld serve as an independent prognostic factor in HCC. This study presents data that supports that RASA1 is a relevant target for personalized cancer treatment in HCC.

\section{Materials and Methods}

Patients. Primary tumor tissues were obtained from 226 HCC patients receiving surgery in Changhua Christian Hospital from July 2011 to November 2013. The patients' relevant clinical data, including demographic information, pathologic diagnosis, laboratory finding and follow up information, were obtained from medical records. All samples and the respective protected health information were acquired in accordance with the protocols approved by the Changhua Christian Hospital Institutional Review Board. The age of all patients was between 29 and 81 years. Clinical patterns and overall survival data were analyzed by chart review. The clinical characteristics of these 226 specimens are shown in Table I. The follow-up period from surgery until death or the endpoint of this study was 884 days and the median overall survival of all patients was 935 days. All samples and the respective protected health information were acquired in accordance with the protocols approved by the Changhua Christian Hospital Institutional Review Board. All procedures performed in studies involving human participants were in accordance with the ethical standards of the institutional and/or national research committee and with the 1964 Helsinki declaration and its later amendments or comparable ethical standards.

Immunohistochemistry (IHC). Immunohistochemistry (IHC) was performed in order to detect RASA1 expression from paraffinembedded liver cancer specimens. The RASA1 antibody (Ab40677)
Table I. Relationship of clinical parameters with RASA1 expression in hepatocellular carcinoma patients.

\begin{tabular}{|c|c|c|c|c|}
\hline \multirow[b]{2}{*}{ Variables } & \multirow[b]{2}{*}{$\mathrm{N}$} & \multicolumn{2}{|c|}{ RASA1 } & \multirow[b]{2}{*}{$p$-Value } \\
\hline & & Low & High & \\
\hline \multicolumn{5}{|l|}{ Age (years) } \\
\hline$<65$ & 124 & $59(48 \%)$ & $65(52 \%)$ & \multirow[t]{2}{*}{0.603} \\
\hline$\geq 65$ & 102 & $45(44 \%)$ & $57(56 \%)$ & \\
\hline \multicolumn{5}{|l|}{ Gender } \\
\hline Female & 61 & $27(44 \%)$ & $34(56 \%)$ & \multirow[t]{2}{*}{0.571} \\
\hline Male & 165 & $80(48 \%)$ & $85(52 \%)$ & \\
\hline \multicolumn{5}{|l|}{ Differentiation } \\
\hline Well & 12 & $4(33 \%)$ & $8(67 \%)$ & \multirow[t]{4}{*}{0.02} \\
\hline Moderate & 108 & $51(47 \%)$ & $57(53 \%)$ & \\
\hline Poor & 96 & $58(60 \%)$ & $38(40 \%)$ & \\
\hline Undifferentiation & 10 & $7(70 \%)$ & $3(30 \%)$ & \\
\hline \multicolumn{5}{|l|}{ Stage } \\
\hline I, II & 181 & $8246 \%)$ & $99(54 \%)$ & \multirow[t]{2}{*}{0.133} \\
\hline III, IV & 45 & $26(58 \%)$ & $19(42 \%)$ & \\
\hline \multicolumn{5}{|c|}{ Hepatitis B surface antigen } \\
\hline Negative & 96 & $56(58 \%)$ & $40(42 \%)$ & \multirow[t]{3}{*}{0.751} \\
\hline Positive & 114 & $69(61 \%)$ & $45(39 \%)$ & \\
\hline Missing & 6 & & & \\
\hline \multicolumn{5}{|l|}{ Hepatitis $C$ virus } \\
\hline Negative & 135 & $61(45 \%)$ & $74(55 \%)$ & \multirow[t]{3}{*}{0.127} \\
\hline Positive & 71 & $40(56 \%)$ & $31(44 \%)$ & \\
\hline Missing & 20 & & & \\
\hline \multicolumn{5}{|l|}{ Tumor number } \\
\hline Single & 179 & $88(49 \%)$ & $91(51 \%)$ & \multirow[t]{2}{*}{0.185} \\
\hline Multiple & 47 & $18(38 \%)$ & $29(62 \%)$ & \\
\hline \multicolumn{5}{|l|}{ Tumor size } \\
\hline$<5 \mathrm{~cm}$ & 145 & $57(39 \%)$ & $88(61 \%)$ & \multirow[t]{2}{*}{0.005} \\
\hline$\geq 5 \mathrm{~cm}$ & 81 & $48(59 \%)$ & $41(41 \%)$ & \\
\hline
\end{tabular}

* $p$-Value was obtained from $\mathrm{X}^{2}$ test.

was purchased from Abcam (Cambridge, USA). The slides were stained with primary antibody using EnVision Detection Systems Peroxidase/DAB, Rabbit/Mouse kit (Dako, Glostrup, Denmark). The slides were photographed with the microscope (BX50, OLYMPUS, Japan, Tokyo). In liver cancer specimens, the detailed scores for IHC were defined as described previously $(16,17)$, and the IHC score of RASA1 for each specimen were defined as the cell staining intensity $(0=$ nil; $1=$ weak; $2=$ moderate; and $3=$ strong) multiplied by the percentage of labeled cells $(0-100 \%)$, leading to scores from 0 to 300 . A score higher than the mean was defined as 'high' expression, while a score equal to or lower than the mean was categorized as 'low' expression in tumor.

Statistical analysis. Scoring of immunoreactivity was compared with clinical data to assess correlation with clinical outcome. Chisquare analysis and paired-samples $t$-test were conducted using SPSS software (Version 13.0 SPSS Inc, Chicago, IL, USA). Differences between experimental groups were calculated with the log-rank test. Survival data was analyzed using the Kaplan-Meier method and variables related to survival were analyzed using SPSS software. Differences with $p$-values of $<0.05$ are considered to be statistically significant. 
A
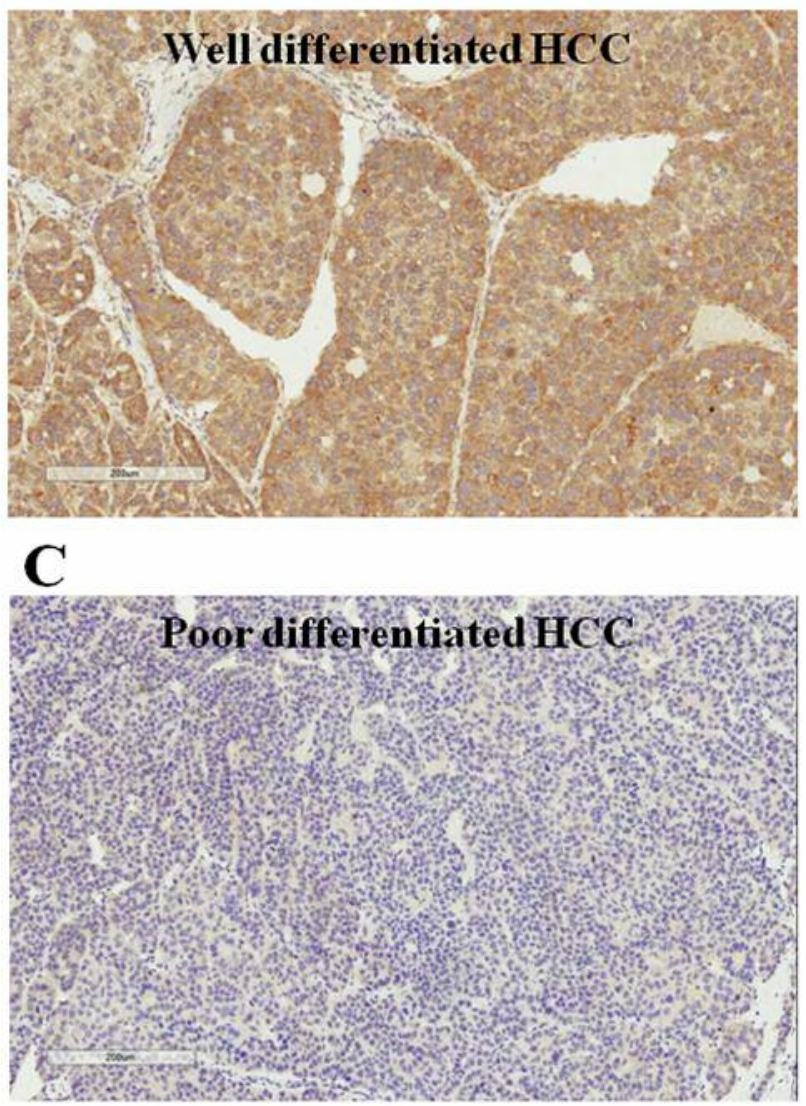

B

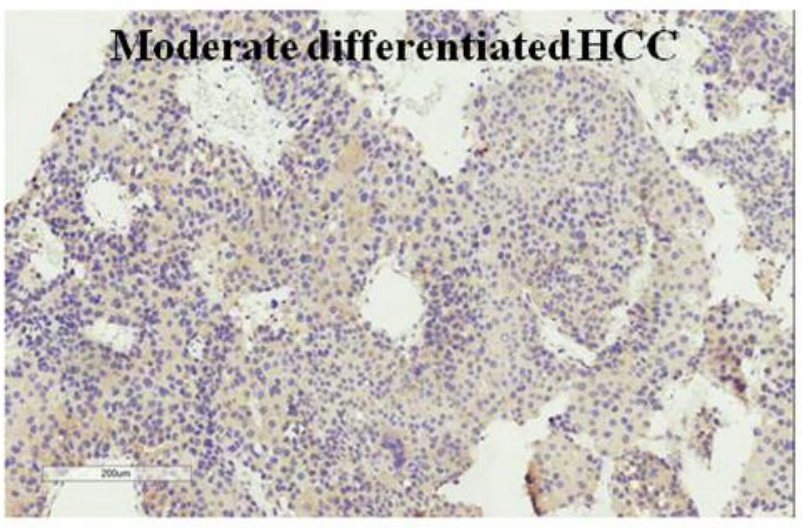

D

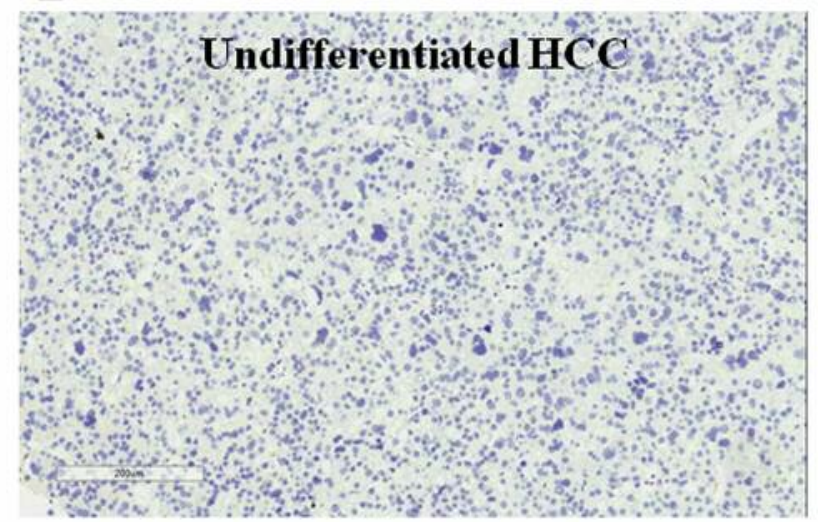

Figure 1. RASA1 protein expression in degree of differentiation in HCC. Positive RASA1 protein expression was predominantly observed in (A) well-differentiated and (B) moderate HCC, but rarely observed in $(C)$ poorly differentiated and $(D)$ undifferentiated HCC.

\section{Results}

RASA1 expression is significantly correlated with differentiation and tumor size. A total of $226 \mathrm{HCC}$ specimens were analyzed in the study. The clinicopathological characteristics of these patients are listed in Table I. Age, gender, stage, Hepatitis B surface antigen (HBV), Hepatitis $\mathrm{C}$ virus (HCV) and the tumor number were not significantly different between patients with low versus high expression level of RASA1. We observed that $38.68 \%$ (41/106) of the high-grade (poorly differentiated/undifferentiated) HCC and $54.17 \%(65 / 120)$ of the low-grade (well/moderate differentiated) HCC expressed high levels of RASA1 protein. The difference between incidence of high RASA1 expression in high- and low-grade HCC was statistically significant $(p=0.02)$. Thus, our data showed that RASA1 protein level was relatively weak in undifferentiated and poorly differentiated tumor samples (Figure 1). Besides, patients with a high level of RASA1 expression had smaller tumor size $(43.59 \pm 36.21 \mathrm{~mm}$ versus $55.25 \pm 41.77 \mathrm{~mm})$ than patients

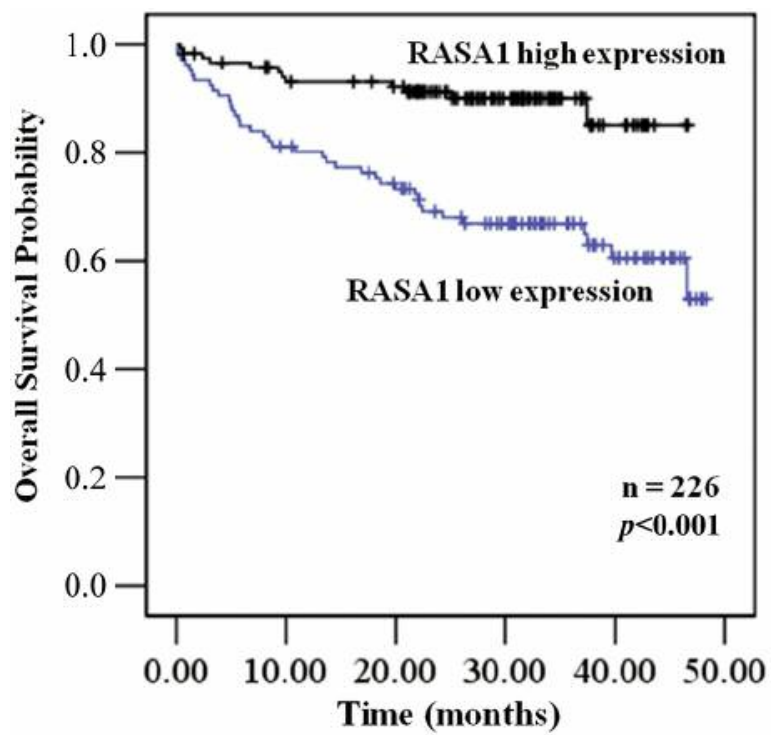

Figure 2. Kaplan Meier plots of the overall survival (OS) rates of HCC patients with high RASA1 expression versus those with low RASAl expression. 
with low level of RASA1 expression. Previous studies have demonstrated that differentiation and tumor size are important predictors of survival that it's potential due to the higher RASA1 expression in the well-differentiated HCC (Figure 1). Therefore, our data suggest that RASA1 expression had a correlation with tumor differentiation and tumor growth and could serve as a prognostic marker for HCC.

Overall survival (OS) according to differentiation, tumorNode-Metastasis (TNM) stage, tumor size and RASAl expression level. The correlation of RASA1 and clinicopathological characteristics with patients' survival was statistically examined by univariate analysis and shown in Table II. Results indicated the significance survival function in several characteristics including age, gender, differentiaiton, TNM stage, hepatitis b surface antigen (HBV), hepatitis c virus (HCV), tumor number, tumor size and RASA1 expression (OS: $p=0.465$ for age, $p=0.478$ for gender, $p=0.038$ for differentiaition, $p<0.001$ for TNM stage, $p=0.903$ for $\mathrm{HBV}, p=0.29$ for $\mathrm{HCV}, p=0.951$ for tumor number, $p<0.001$ for tumor size and $p<0.001$ for RASA1 expression). First, our analysis of clinical samples revealed that 120 patients with low-grade (well- and moderatedifferentiation) had longer survival time than 106 patients with high-grade (poor- and un-differentiation). The OS of HCC patients with low-grade and high-grade was 30.69 \pm 9.88 and $26.53 \pm 12.66$ months, respectively $(p<0.038)$. Next, our data also showed that the OS of HCC patients with tumor size $<5 \mathrm{~cm}$ and $\geq 5 \mathrm{~cm}$ was $31.6 \pm 8.96$ and $23.81 \pm 14.06$ months, respectively $(p<0.001)$ and HCC patients with stage III/IV had a worse prognosis than those with stage I/II specimens $(p<0.001)$. Futhermore, we examined whether RASA1 expression is associated with clinical outcomes in HCC patients. Kaplan-Meier survival analysis showed that patients with low level of RASA1 expression correlated with a significantly poorer survival than those with high level of RASA1 expression (Figure 2). These results suggest that RASA1 expression is lower in the high-grade than the lowgrade and may be correlated with tumor growth and consequently result in poor outcomes in HCC patients.

\section{Discussion}

RASA1 is ubiquitously expressed across different tissue types. Although previous studies revealed that RASA1 may play tumor suppressive roles in many cancer types $(12,14$, 15), the correlation between the role of RASA 1 expression, tumor differentiation and overall survival in HCC have not been previously addressed in detail. This study shows that RASA 1 protein is rarely expressed in HCC, especially in poor differentiated and undifferentiated tumors or large-sized tumors. Previous studies have demonstrated that differentiation and tumor size are important predictors of
Table II. Univariate analysis of influence of clinical characteristics on overall survival in hepatocellular patients.

\begin{tabular}{|c|c|c|c|c|}
\hline \multirow[b]{2}{*}{ Characteristics } & \multirow[b]{2}{*}{$\mathrm{N}$} & \multicolumn{2}{|c|}{ Overall survival (OS) } & \multirow[b]{2}{*}{ Log-rank } \\
\hline & & $\begin{array}{l}\text { Median } \\
\text { survival }\end{array}$ & $\begin{array}{c}\text { Survival } \\
\text { (months) }(\%)\end{array}$ & \\
\hline \multicolumn{5}{|l|}{ Age (years) } \\
\hline$<65$ & 124 & 30.8 & $79.37 \%$ & 0.465 \\
\hline$\geq 65$ & 102 & 29.13 & $76.00 \%$ & \\
\hline \multicolumn{5}{|l|}{ Gender } \\
\hline Female & 61 & 28.07 & $75.00 \%$ & 0.478 \\
\hline Male & 165 & 30.6 & $78.92 \%$ & \\
\hline \multicolumn{5}{|l|}{ Differentiation } \\
\hline Well, Moderate & 120 & 30.6 & $88.39 \%$ & 0.038 \\
\hline Poor, Undifferentiation & 106 & 26.5 & $67.26 \%$ & \\
\hline \multicolumn{5}{|l|}{ Stage } \\
\hline I, II & 181 & 31.23 & 84.43 & $<0.001$ \\
\hline III, IV & 45 & 22.13 & $57.50 \%$ & \\
\hline \multicolumn{5}{|l|}{ Hepatitis B surface antigen } \\
\hline Negative & 96 & 29.83 & $77.08 \%$ & 0.903 \\
\hline Positive & 114 & 30.87 & $76.79 \%$ & \\
\hline \multicolumn{5}{|l|}{ Hepatitis $\mathrm{C}$ virus } \\
\hline Negative & 135 & 31.63 & $72.48 \%$ & 0.29 \\
\hline Positive & 71 & 32.2 & $78.85 \%$ & \\
\hline \multicolumn{5}{|l|}{ Tumor number } \\
\hline Single & 179 & 31.17 & $77.78 \%$ & 0.951 \\
\hline Multiple & 47 & 25.4 & $78.38 \%$ & \\
\hline \multicolumn{5}{|l|}{ Tumor size } \\
\hline$<5 \mathrm{~cm}$ & 145 & 31.6 & $86.39 \%$ & $<0.001$ \\
\hline$\geq 5 \mathrm{~cm}$ & 81 & 23.8 & $62.03 \%$ & \\
\hline \multicolumn{5}{|l|}{ RASA1 } \\
\hline Low & 106 & 30.6 & $64.15 \%$ & $<0.001$ \\
\hline High & 120 & 29.5 & $89.92 \%$ & \\
\hline
\end{tabular}

* $p$-Value was obtained from $\mathrm{X}^{2}$ test.

survival (18-20) that it's potential due to the higher RASA1 expression in the well-differentiated HCC (Figure 1). RASA1 protein expression was inversely associated with liver cancer aggressiveness (Figure 1). Additionally, RASA1 levels decreased significantly in the nodules of tumor size $\geq 5 \mathrm{~cm}$ compared to their corresponding smaller nodules (Table I). As tumor size increased, survival progressively worsened. Despite accumulating evidence pointing to the regulatory role of RASA1 in cancer progression and proliferation, its regulatory role in outcomes of HCC is still poorly understood. Therefore, our results showed that reduced RASA1 expression is significantly correlated with poor outcome in HCC (Figure 2).

A previous study indicated that miR-182 could be induced in HCC cells under hypoxia and promote angiogenesis by targeting RASA1 (15). In another study, it was shown that onco-miRNA, miR-21, may promotes malignant behaviors of cancer cells through regulation of RASA1 expression (14). Moreover, a recent study reported that PTP1B de- 
phosphorylates PITX1 and weakens its protein stability and the transcriptional activity for RASA1 expression, resulting in enhanced tumor suppression in human liver cancer cells (12). Taken together, these data may suggest RASA1 not only regulates cell apoptosis, cell proliferation and tumor growth, but also stimulates cell invasion and cancer metastasis. Our finding in this study is in agreement with studies in several other of cancer, suggesting that RASA1 may act tumor suppressor.

In conclusion, our findings reveal that RASA1 expression level could confer poor outcome and potentially serve as one of prognosis biomarkers and targets for intervention of tumorigenesis in HCC.

\section{Conflicts of Interest}

The Authors declare that they have no conflict of interest.

\section{Acknowledgements}

This study was funded by grants 102-2321-B-750-001- and 1032314-B-442-002-MY3 from the Ministry of Science and Technology, Taiwan, and RB15001 and RB16001 from Show Chwan Memorial Hospital, Taiwan.

\section{References}

1 Llovet JM, Zucman-Rossi J, Pikarsky E, Sangro B, Schwartz M, Sherman M and Gores G: Hepatocellular carcinoma. Nat Rev Dis Primers 2: 16018, 2016.

2 Jarcuska P, Drazilova S, Fedacko J, Pella D and Janicko M: Association between hepatitis $\mathrm{b}$ and metabolic syndrome: Current state of the art. World J Gastroenterol 22(1): 155-164, 2016.

3 El-Serag HB: Hepatocellular carcinoma. N Engl J Med 365(12): 1118-1127, 2011.

4 Filchtinski D, Sharabi O, Rüppel A, Vetter IR, Herrmann C and Shifman JM: What makes ras an efficient molecular switch: A computational, biophysical, and structural study of ras-gdp interactions with mutants of raf. J Mol Biol 399(3): 422-435, 2010.

5 Bourne HR, Sanders DA and McCormick F: The gtpase superfamily: A conserved switch for diverse cell functions. Nature 348(6297): 125-132, 1990.

6 McLaughlin Sara K, Olsen Sarah N, Dake B, De Raedt T, Lim E, Bronson Roderick T, Beroukhim R, Polyak K, Brown M, Kuperwasser C and Cichowski K: The rasgap gene, rasal2, is a tumor and metastasis suppressor. Cancer Cell 24(3): 365-378, 2013.

7 Bourne HR, Sanders DA and McCormick F: The gtpase superfamily: Conserved structure and molecular mechanism. Nature 349(6305): 117-127, 1991.

8 Tabrizian P, Jibara G, Shrager B, Schwartz M and Roayaie S: Recurrence of hepatocellular cancer after resection: Patterns, treatments, and prognosis. Ann Surg 261(5): 947-955, 2015.
9 Huss WJ, Gray DR, Greenberg NM, Mohler JL and Smith GJ: Breast cancer resistance protein-mediated efflux of androgen in putative benign and malignant prostate stem cells. Cancer Res 65(15): 6640-6650, 2005.

10 Hui K, Wu K, Fan J and He D: Mp45-20 rasal2, a novel ras gtpase activating protein, inhibits stemness and epithelialmesenchymal transition via mapk/sox 2 pathway in bladder cancer. J Urol 195(4): e616, 2016.

11 Chen S, Do JT, Zhang Q, Yao S, Yan F, Peters EC, Schöler HR, Schultz PG and Ding S: Self-renewal of embryonic stem cells by a small molecule. Proc Natl Acad Sci 103(46): 17266-17271, 2006.

12 Tai WT, Chen YL, Chu PY, Chen LJ, Hung MH, Shiau CW, Huang JW, Tsai MH and Chen KF: Protein tyrosine phosphatase $1 \mathrm{~b}$ dephosphorylates pitx1 and regulates p120rasgap in hepatocellular carcinoma. Hepatology 63(5): 1528-1543, 2016.

13 Maertens $\mathrm{O}$ and Cichowski K: An expanding role for ras gtpase activating proteins (ras gaps) in cancer. Adv Biol Regul 55: 114, 2014.

14 Zhang L, Zhan X, Yan D and Wang Z: Circulating microrna-21 is involved in lymph node metastasis in cervical cancer by targeting rasa1. Int J Gynecol Cancer 26(5): 810-816, 2016.

15 Du C, Weng X, Hu W, Lv Z, Xiao H, Ding C, Gyabaah O-aK, Xie H, Zhou L, Wu J and Zheng S: Hypoxia-inducible mir-182 promotes angiogenesis by targeting rasa1 in hepatocellular carcinoma. J Exp Clin Cancer Res 34(1): 67, 2015.

16 Yu HC, Hung MH, Chen YL, Chu PY, Wang CY, Chao TT, Liu CY, Shiau CW and Chen KF: Erlotinib derivative inhibits hepatocellular carcinoma by targeting cip2a to reactivate protein phosphatase 2a. Cell Death Dis 5: e1359, 2014.

17 Chen YL, Chen PM, Lin PY, Hsiau YT and Chu PY: Abcg2 overexpression confers poor outcomes in hepatocellular carcinoma of elderly patients. Anticancer Res 36(6): 2983-2988, 2016.

18 Han DH, Choi GH, Kim KS, Choi JS, Park YN, Kim SU, Park JY, Ahn SH and Han K-H: Prognostic significance of the worst grade in hepatocellular carcinoma with heterogeneous histologic grades of differentiation. J Gastroenterol Hepatol 28(8): 13841390, 2013.

19 Liang P, Dong B, Yu X, Yu D, Wang Y, Feng L and Xiao Q: Prognostic factors for survival in patients with hepatocellular carcinoma after percutaneous microwave ablation. Radiology 235(1): 299-307, 2005.

20 Narod SA: Tumour size predicts long-term survival among women with lymph node-positive breast cancer. Curr Oncol 19(5): 249-253, 2012.
Received December 1, 2016

Revised December 23, 2016

Accepted December 29, 2016 